\title{
Pharmacology Shared Resource
}

National Cancer Institute

\section{Source}

National Cancer Institute. Pharmacology Shared Resource. NCI Thesaurus. Code C39494.

The Pharmacology Shared Resource provides Cancer Center investigators conducting preclinical and clinical studies with analytical and technical pharmacology support in the areas of sample handling and tracking, pharmacokinetic modeling, drug-drug interaction, pharmacodynamics, drug assays, in vitro toxicity assays and molecular analysis of tumor tissue; human safety issues, adverse reactions risk evaluation and management, chemotherapy monitoring; assistance in study design, optimal sampling techniques and data interpretation. 